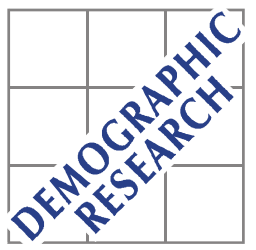

Demographic Research a free, expedited, online journal of peer-reviewed research and commentary in the population sciences published by the Max Planck Institute for Demographic Research Konrad-Zuse Str. 1, D-18057 Rostock · GERMANY www.demographic-research.org

DEMOGRAPHIC RESEARCH

VOLUME 14, ARTICLE 10, PAGES 179-216

PUBLISHED 10 MARCH 2006

http://www.demographic-research.org/Volumes/Vol14/10/

DOI: 10.4054/DemRes.2006.14.10

Research Article

Race/ethnic differences and age-variation in the effects of birth outcomes on infant mortality in the U.S.

Daniel A. Powers

W. Parker Frisbie

Robert A. Hummer

Starling G. Pullum

Patricio Solis

(C) 2006 Max-Planck-Gesellschaft. 


\section{Table of Contents}

$1 \quad$ Introduction $\quad 180$

1.1 Race/ethnic variation in pregnancy outcomes 181

$\begin{array}{lll}1.2 & \text { Birth outcome measures } & 182\end{array}$

1.2.1 Objectives of this research 184

2 Data and methods $\quad 185$

$\begin{array}{lll}2.1 & \text { Data } & 185\end{array}$

2.2 Methods 186

2.3 Birth outcome measures 186

2.4 Modeling infant mortality 189

$\begin{array}{lll}2.5 & \text { Model specification } & 190\end{array}$

3 Results 191

3.1 Race/ethnic variation in age-specific mortality rates 191

3.2 Multivariate analysis 193

3.2.1 Static birth outcome effects model 193

3.2.2 Age-varying Birth Outcome Effects Model 194

3.2.3 Description of birth outcome effects 196

4 Discussion 201

5 Acknowledgements 204

$\begin{array}{ll}\text { References } & 205\end{array}$

Appendix A 211

Race/Ethnic Specific Birth Weight Optima 211

Further Results on Age-Varying Effects 212 


\title{
Race/ethnic differences and age-variation in the effects of birth outcomes on infant mortality in the U.S.
}

\author{
Daniel A. Powers ${ }^{1}$ \\ W. Parker Frisbie ${ }^{2}$ \\ Robert A. Hummer ${ }^{3}$ \\ Starling G. Pullum ${ }^{4}$ \\ Patricio Solis ${ }^{5}$
}

\begin{abstract}
This paper investigates effects of continuous measures of birth outcomes on U.S. infant mortality for non-Hispanic white, black, and Mexican-American females. Birth outcomes show larger effects on neonatal mortality with smaller effects later on. Unlike static effects models, age-varying effect models of early and late gestational age and small birth weight statistically adjust for the black neonatal mortality disadvantage. While Mexican American infant mortality largely mirrors that of whites throughout the first year, higher neonatal mortality among blacks reflects disadvantaged birth outcome composition. Higher postneonatal mortality among blacks remains, net of birth outcomes, and may largely reflect socio-environmental disadvantages.
\end{abstract}

\footnotetext{
${ }^{1}$ Direct all correspondence to Daniel A. Powers, Department of Sociology, 1 University Station, A1700, University of Texas at Austin, Austin,

TX 78712, USA. E-mail: dpowers@mail.la.utexas.edu

${ }^{2}$ University of Texas at Austin. E-mail: frisbie@prc.utexas.edu

${ }^{3}$ University of Texas at Austin. E-mail: hummer@prc.utexas.edu

${ }^{4}$ University of Texas at Austin. E-mail: starling@prc.utexas.edu

${ }^{5}$ El Colegio de México. E-mail: psolis@colmex.mx
} 


\section{Introduction}

Demographic, clinical, and epidemiological research consistently documents the greatly increased risk of mortality among preterm and low birth weight infants (Cramer 1987; Frisbie, Forbes, and Hummer 1998; Hack et al. 1995; Kiely and Susser 1992; Kline, Stein, and Susser 1989; McCormick 1985). Several relatively recent demographic studies (Frisbie, Forbes and Pullum 1996; Frisbie et al. 1997; Hummer et al. 1999) have considered alternative specifications that make finer-grained distinctions than do the conventional dichotomous classifications of birth weight and gestational age. These alternative categorizations of birth outcomes follow in a long tradition (cf. Yerushalmy 1967) and have proven useful in more precisely distinguishing higher-risk from lowerrisk infants. However, while typologies constructed by categorizing continuous data may be theoretically justified and yield meaningful results, they inevitably discard potentially important information.

Retaining greater detail and precision in birth outcome measurement would seem to be increasingly important for the study of race/ethnic differentials in infant mortality because of historic changes in the structure of infant mortality that have occurred in the past two decades (Gortmaker and Wise 1997; Wise 2003). There seems little doubt that technological innovations and general improvement in perinatal care have produced a situation in which "the greatest progress in enhancing infant survival occurred in low birth weight and preterm infants" (Alexander et al. 1999b: 71; see also Gortmaker and Wise 1997 and Wise 2003). But, despite substantial general reductions in absolute levels of infant mortality in the United States, the relative black-white disparity in risk of infant death has been increasing (Guyer et al. 1998; Minono et al. 2002). In this context, there is a renewed interest in measurement strategies that retain the continuous metric of birth outcomes (Solis, Pullum, and Frisbie 2000) within meaningful subgroups of gestational age, as suggested long ago by Yerushalmy (1967) and reinforced in more contemporary public health research (Wilcox 2003; Wilcox and Skjœrven 1992). However, a potential problem with all of the aforementioned research is that birth outcome effects are modeled without regard to the widely differing risks of mortality that are highest during the first day of life and decrease throughout the first year (Mathews et al. 2002).

The general purpose of this paper is to apply recent developments in the measurement of birth outcomes to: (1) determine the extent to which important components of birth outcomes (birth weight and gestational age) influence infant mortality risk, (2) to examine age-variation (i.e., nonproportionality) in birth outcome effects, and (3) to assess race/ethnic variation in this dynamic. This paper is organized as follows: Section 1 provides background on issues motivating this investigation, namely race/ethnic disparities in survival and measurement of birth outcomes; Section 
2 discusses data and the statistical models and methods applied to assess birth outcome effects; and Section 3 presents results from this study. Further implications of this research are discussed in Section 4.

\subsection{Race/ethnic variation in pregnancy outcomes}

The motivation for studies of pregnancy outcomes ${ }^{6}$ that are comparative by race/ethnicity is well known. For many years, the greatest concern has been with blackwhite disparities in that, as far into the past as data exist, the black infant mortality rate has been close to double that of the white rate, and, over the past decade or two, the relative disparity has actually increased up to about 2.4 (Buehler et al. 1987; Guyer et al. 1998; Hoyert et al. 2001; Hummer 1996; Minono et al. 2002). The higher black infant mortality rate is generally attributed to higher mortality at normal birth weights and higher rates of low birth weight (where mortality is higher). However, it is also well documented that blacks face lower birth weight specific mortality at low birth weights (Buehler et al. 1987; Wilcox and Russell 1990), leading to the so-called "pediatric paradox."

More recently, interest in Hispanic, especially Mexican American, infant mortality has burgeoned, fueled by the consistent finding that the Mexican American infant mortality rate is remarkably similar to the rate among non-Hispanic whites, despite the fact that the former group is quite disadvantaged with respect to socioeconomic status and access to, and/or utilization of, health services (Albrecht et al. 1996; Becerra et al. 1991; Frisbie et al. 1998; Frisbie and Song 2003; Hummer et al. 1999; Rumbaut and Weeks 1991; 1996; Scribner 1996). For example, Mexican Americans, on average, have lower levels of education, less access to prenatal care, and are less apt to have health insurance of any kind than either non-Hispanic blacks or non-Hispanic whites (Frisbie et al. 1997). Reasons for the seemingly paradoxical coupling of relatively positive pregnancy outcomes with a disadvantageous risk profile include positive selection of migration (Frisbie 2005; Palloni and Morenoff 2001) and culturally-based behavioral buffers to morbidity and mortality (Cobas et al. 1996; Guendelman and Abrams 1995; Scribner 1996; Scribner and Dwyer 1989; Rumbaut and Weeks 1991, 1996).

${ }^{6}$ We use the term "pregnancy outcomes" to refer to both infant death/survival and to birth outcomes (birth weight and gestational age). 


\subsection{Birth outcome measures}

Categorical measures of birth outcomes have generally received the most attention. Yerushalmy (1967) argued that classification systems should combine birth weight and gestational age into relevant categories. His typology consisted of five categories ranging from very low birth weight $(<1500$ grams) to moderately low weight (1500 to 2499 grams) to normal weight ( $>2500$ grams) births, with gestational age categories ( $<37$ weeks versus $37+$ weeks) dichotomizing the latter two categories. Demographers have employed a less extensive classification for some analyses in which low birth weight (LBW) infants are divided into premature births ( $<37$ weeks gestation and < 2500 grams) and those who are intrauterine growth retarded (37 or more weeks gestation and < 2500 grams), with all births weighing 2500 grams or more classified as normal (Kallan 1993; Hummer et al. 1995). More recent modifications proposed by Frisbie et al. $(1996,1997)$ distinguish heavy premature infants ( $<37$ weeks, $\geq 2500$ grams) and also incorporate the fetal growth ratio (Kramer et al. 1988, 1989) as a means of identifying immature births that would not be detected using conventional measures.

In many respects, these classifications were created to reflect specific analytic problems and interests of researchers. To the extent that they simplify analysis and interpretation of patterns of association, there is much to recommend their use. But, as alluded to previously, categorical measures are limited in important ways. First, categories inherently involve fixed cutoff points in the continuous distribution of birth outcomes and thus create an image of discontinuity in the association between birth outcomes and infant mortality. Second, significant variation within categories is ignored, thus implying homogeneity of risk within categories of birth outcomes. Solis et al. (2000) note the obvious problem that arises when conventional birth outcome dichotomies are employed (i.e., a cut-point of 37 weeks gestation to distinguish preterm from term births and 2500 grams as the cut-point distinguishing low from normal weight births). For example, an infant born at 36 weeks gestation is treated as having the same risk of death as one born at 30 weeks, and as being at much greater risk of death than one born at 37 weeks - at best, a gross oversimplification. Perhaps less often recognized, the optimal birth weight for survival is far higher than 2500 grams, and births at weights exceeding the optimal point incur a somewhat increased risk of infant mortality (cf. Alexander et al. 1999a; Myers and Ferguson 1989; Wilcox and Russell 1983). Following Wilcox and Skjœrven (1992), Solis et al. (2000) advocated standardizing birth weight to a gestational age-relative scale to simultaneously compare gestational age and birth weight effects on infant mortality. This procedure allows the partial dependence of birth weight on gestational age to be taken into account. It departs from a categorical scheme by including birth outcomes as continuous variables. These authors also utilized measures that reflect deviations from optimal combinations 
of birth weight and gestational age that yield the lowest levels of infant mortality, and modeled both main and interaction effects of these birth outcome dimensions, thus allowing a more precise evaluation of the relationship of each birth outcome with infant mortality risk.

Recent efforts to improve measurement of birth outcomes have complemented, and to some extent arisen from, unresolved issues regarding the relationship between birth outcomes and infant mortality risk. Therefore, it is of considerable interest to determine whether recent alternative measurement approaches permit us to learn more about race/ethnic disparities in infant mortality. Another issue involves the debate over the causal significance of birth weight. Contrary to the conclusion reached in the vast majority of literature on the subject, Wilcox (2001a, 2001b) has argued that the association between birth weight and infant mortality is non-causal. If this interpretation is correct, then "(e)limination of LBW (low birth weight) is neither practical nor necessary in order to achieve the lowest possible rates of infant mortality" (Wilcox 2001a: 1238). While there have been challenges to the Wilcox perspective (David 2001; Hertz-Picciotto 2001), the recent debate is more than a little reminiscent of an earlier concern, expressed primarily in the public health literature, that birth weight and gestational age play somewhat separate roles in infant survival, but unfortunately, "attention has been diverted from the task of establishing the independent effects of gestational age and size" (Kline et al. 1989: 173; see also Yerushalmy 1967 and Battaglia and Lubchenco 1967).

Wilcox's causal argument may be correct insofar as the actual mass of the infant does not generate morbidity and mortality. Using a series of examples, Wilcox demonstrates that percent low birth weight (LBW) (birth weights < 2500g) alone is not a good surrogate indicator of a population's infant risk, as it largely reflects a leftward shift in the predominant distribution of birth weight. By standardizing birth weight to a relative weight scale ( $z$-scale), population differences can be more readily observed. The critical determinant of mortality differences are population differences in the residual distributions (percentage of births that are both small and preterm). These differences are difficult to detect using absolute measures like LBW or unstandardized birth weight. Critics have countered that birth weight is informative about population health, but that challenges remain to understand: "(1) the genetic and environmental influences on birth weight, and (2) distinguish when reduced birth weight is and when it is not prognostic of poorer health and development." (HertzPicciotto 2001: 1244). As Wise (2003) suggests, it is perhaps best to view birth weight and gestational age as outcomes of complex pathophysiologic processes. From this perspective, one can retain the proxy status of these measures while avoiding reification of birth outcomes. 
A potentially serious shortcoming of much research in this area is the failure to systematically consider models that allow age variation in the effects of birth weight and gestational age on infant mortality risk. ${ }^{7}$ A disproportionate number of all infant deaths in the United States occur in the neonatal period (deaths prior to the 28th day of life), and a majority of neonatal deaths occur within the first week (Alexander et al. 1999b), with considerable concentration in the first day (Preston et al. 2001). Although numerous studies have focused separately on the effects of birth outcomes on neonatal (deaths to infants $<28$ days of life) and postneonatal mortality (deaths from the 28th day through the remainder of the first year), potentially important sources of age variation in the neonatal period are often ignored. Further, it may be that both the main and conjoint effects of birth weight and gestational age interact with infant age in influencing mortality risk throughout the first year of life.

\subsubsection{Objectives of this research}

In general, we are interested in the extent to which birth weight and gestational age influence infant mortality risk and the extent to which race/ethnic variation exists in this relationship. More specifically, the objectives of the present research are to: (1) apply alternative measurement strategies that preserve the continuous metric of birth weight and gestational age in the modeling of the relationship between birth outcomes and infant mortality risk based on recent data available for the nation as whole, (2) construct models for non-Hispanic blacks and Mexican Americans, as well as for non-Hispanic whites, based on race/ethnic-specific birth weight distributions, and (3) model the agespecific mortality rate and assess the assumption of proportionality in the effects of birth outcomes on infant mortality. (4) Finally, since our approach incorporates both the main and conjoint effects of gestational age and birth weight, the results should allow some additional insight into the question of the relative magnitude of each on infant mortality risk.

\footnotetext{
${ }^{7}$ A notable exception is the work of Platt et al. (2004), who model perinatal mortality risk using proportional hazards models on a gestational age time scale, and include time-varying effects of race, maternal smoking, and birth as time-varying covariate.
} 


\section{Data and methods}

\subsection{Data}

The data employed are the National Center for Health Statistics (NCHS) linked birth/infant death cohort files for 1995-1998, which include all infants born alive in the U.S. during those years and mortality information for the subset of infants in the cohorts who died during the first year of life. The data set consists of approximately four million cases each year, and the match rate is exceptional-as early as 1989, more than $97 \%$ of death records were successfully linked to a birth certificate (U.S. Department of Health and Human Services 1995). Also beginning in 1989, a clinical estimate of gestational age was included, which NCHS uses, along with other diagnostic techniques, to arrive at more accurate recording of gestational age than is possible by the conventional method of subtracting date of birth from date of last normal menses. As recommended by NCHS, mother's race/ethnicity is used to distinguish non-Hispanic Whites (hereafter, "Whites"), non-Hispanic Blacks (hereafter "Blacks") and Mexican Americans.

There were 15,627,407 linked birth/infant death records for the years 1995-1998. We excluded male infants, plural births, mothers who were nonresidents of the country, and records missing birth weight or gestational age. We also excluded a small number of births with gestational age under 22 weeks or birth weight under 500 grams, both because of the increased probability that these figures were incorrectly recorded and because of the possibility that these were misclassified stillbirths. We further restricted the analysis to infants born to white, black, and Mexican origin women; all other race/ethnic groups added together had fewer births than any of these three groups. These selections resulted in 4,512,567 female births to white women (3.8\% of original records were excluded), of whom 17,395 died; 1,102,235 female births to black women (5.0\% were excluded), of whom 8,757 died; and 926,864 female births to Mexican origin women ( $4.8 \%$ were excluded), of whom 3,589 died. Following Solis et al. (2000) we focus only on females. On average males are born heavier than females, but have higher infant mortality. This is a well known finding that parallels the black-white pediatric paradox. Comparison of age-variation in birth outcome effects by sex and race/ethnicity is an important area for future research, but will necessarily require investigation into race and sex specific optima. One paper (Echeverria-Cruz 2004) has pursued this issue. A thorough analysis of race and sex differences is beyond the scope of this present paper, which serves mainly to extend the work of Solis et al. (2000) to encompass age-variation in risk associated with birth weight and gestational age. 


\subsection{Methods}

We incorporate recent developments in the continuous measurement of birth outcomes as advocated by Solis et al. (2000) and others (e.g., Wilcox and Skjœrven 1992), in which birth outcomes are measured as deviations from optimal combinations of birth weight and gestational age for infant survival. However, we extend this general approach in important ways. First, we broaden the analysis to include non-Hispanic blacks and Mexican Americans-populations that have rarely been previously analyzed in this manner. In so doing, we highlight race and ethnic differences in the effects of birth outcomes on infant mortality using recent data for these populations. Further, we estimate proportional hazard models of infant mortality that allow effects of birth outcomes on the risk of infant death to vary by age of death during the first year of life, thus allowing for nonproportional effects. Proportional hazards models with agevarying effects permit a more rigorous assessment of the differential impact of continuously measured birth outcomes, and their interactions, on age-specific infant mortality than do the more widely-used static models that assume constant effects throughout the first year of life. We define age intervals to broadly distinguish between mortality in the neonatal and postneonatal period, and also within finer subdivisions (within the 1st day, 1 to 7 days, and 7 to 28 days) in the neonatal period.

\subsection{Birth outcome measures}

The predictors in our multivariate analyses are those used by Solis et al. (2000), who consider two birth outcome measures based on departures from survival optimums. The survival optimum is a point in the birth weight distribution that yields the lowest infant mortality. Solis et al. estimated this optimum to be approximately one standard deviation above the gestational age-specific mean birth weight by examining the IMR as a function of birth weight standardized on a $z$-score scale for births with gestations of 39-41 weeks (about $62 \%$ of all female births among whites). The lowest observed IMR for gestational ages in this range occurred between 1 and 2 standard deviations above the mean birth weight in this range. The one standard deviation criterion for the optimum reflects a lower bound on birth weight (in standardized units) that maximizes survival for gestations of 39-41 weeks. Although this measure lacks a strict biological basis insofar as it cannot account for the etiological pathways leading to departures from optimal birth weight, it is empirically grounded in the sense that it yields the lowest average IMR over a specific range of gestational age. The approach is very similar to that used by Adams et al. (1991) to study perinatal mortality. Support for this approach when comparing different populations stems from the assumption that 
differences between the mean birth weight and the optimum birth weight are nearly the same regardless of race (Chung 1967). The Appendix to this paper provides additional details about the birth weight optimum obtained by Solis et al. (2000), in addition to evidence that one standard deviation above the gestational age specific mean is a reasonable survival optimum birth weight for white and black females using the 19951998 data. Our preliminary results suggest that the survival optimum for Mexican American females is somewhat closer to the gestational age-specific mean birth weight.

In the present context, where race/ethnic differences in effects of birth outcomes are the focus, this standardization approach allows the comparison of mortality for infants of the same relative weight (i.e., relative to the race/ethnic specific distribution of birth weight). When gestational age-specific standardization is used, this identifies infants that are large or small for their gestational age and permits a comparison to infants of the same relative sizes at other gestational ages (Wilcox and Skjœrven 1992). Although Wilcox and Russell proposed that gestational age birth weight standardization be used, they argued that comparisons of populations based on a single standardization were biased. Instead, they argued in favor of the use of population-specific standards (Wilcox and Russell 1983). There is considerable debate in the epidemiological literature on the use of population-specific standards in the study of infant mortality. Population-specific standards have been criticized because they may obscure unexplained population differences (Wise and Pursley 1992). If this is the case, then the use of race/ethnic-specific distributional standards makes comparisons of birth outcome effects across races more problematic insofar as we do not know to what extent observed race/ethnic differences are due to distributional differences or differences in the effects of birth outcomes. However, as Wilcox and Russell (1983) and others have argued, standardization by conventional means, such as using the white birth weight distribution or another single standard, will tend to obscure race/ethnic differences, and lead to comparisons that are biased against the population with heavier birth weights. Platt et al. (2004a) have cautioned that the (race/ethnic specific) relative birth weight standardization approach may create analytic problems due to confounding by gestational age. However, these authors have not addressed possible bias associated with the approach of Wilcox and Skjœrven 1992, which makes use of gestationspecific standardization (i.e., relative birth weights constructed for each gestational age stratum), and may better isolate the effects of birth weight and gestational age than would a standardization based on birth weight alone. ${ }^{8}$ As Adams et al. (1991) have argued, when population standards are used without an accounting of gestational age, analysis may not detect important race/ethnic differences such as the relative survival advantage of black infants at short gestations. Our purpose here is not to bring closure ${ }^{8}$ Although Wilcox and Skjœrven (1992) did not carry out race/ethnic comparisons using this approach, this was pursued in later research by
Adams et al. (1991). 
to this debate but to show the relevance of modeling age-variation in the effects of components of infant mortality risk that reflect the main and conjoint effects of birth weight and gestational age. We next provide the details about the construction of these birth outcome measures.

For each race/ethnic group, a measure $S$ (small) is defined as the difference between one-standard deviation $\left(\sigma_{j}\right)$ above the mean birth weight at gestational age $j$ $\left(\mu_{j}\right)$ and the $i$ th infant's birth weight $\left(W_{i}\right)$, which corresponds to a negative deviation from optimum. More formally,

$$
S_{i}=\frac{\left(\mu_{j}+\sigma_{j}\right)-W_{i}}{\sigma_{j}}=1-Z_{i}
$$

where $Z_{i}$ is the $i$ th infant's standardized birth weight score based on the gestational agespecific mean and standard deviation. This measure is positive when $1-Z_{i}>0$ and 0 otherwise. This measure can be interpreted as the additive effect on the mortality risk due to a decrease in birth weight beyond the gestational age-specific optimum. This is analogous to Wilcox and Russell's (1983) formulation of "risk from low birth weight," $\mathrm{R}_{1}$. As noted in their paper, the relationship between birth weight and mortality follows the form of a dose-response curve, which is "widely used in biological assay to describe mortality from an increasing and potentially lethal dose." (Wilcox and Russell 1983:323). Similarly, an infant is classified on a continuum of heavy birth weight $(H)$ if birth weight exceeds the gestational age-specific optimum, defined as the difference between the $i$ th infant's birth weight and one standard deviation above the gestational age specific mean, or

$$
H_{i}=\frac{W_{i}-\left(\mu_{j}+\sigma_{j}\right)}{\sigma_{j}}=Z_{i}-1
$$

where $Z_{i}$ is the standardized score defined above. This measure is positive when $Z_{i}-1$ $>0$ and 0 otherwise. ${ }^{9}$ This term is analogous to Wilcox and Russell's (1983) measure of "risk from high birth weight," $\mathrm{R}_{2}$, and is meant to capture the increase in risk associated with increases in birth weight beyond the gestational age-specific optimum. The measures $S$ and $H$ are calculated separately for each race/ethnic group, thus giving race/ethnic specific standardizations. This construction implicitly recognizes that gestational age and birth weight may vary together in different ways for different racial and ethnic groups depending, for example, on social context. The population parameters

\footnotetext{
${ }^{9}$ As noted by Wilcox and Russell 1983 and others, birth weight distributions are essentially normal except for overrepresentation in the upper and lower tails.
} 
underlying the standardization are provided in the Appendix (Table A1). Together these terms comprise the birth weight risk components.

In addition to birth weight risk components, we also consider variables that capture the gestational age component of the risk. The variable $E$ (early) is defined in terms of positive deviations (in weeks) below the optimal minimum of 39 weeks of gestation. This captures the increased risk associated with preterm birth. The variable $L$ (late) measures gestational age risk associated with births later than the 41st week. More formally, if $A$ denotes an infant's gestational age in weeks,

$$
E_{i}=39-A_{i} \text {, if } A_{i}<39 \text {, and } 0 \text { otherwise, }
$$

and

$$
L_{i}=A_{i}-41 \text {, if } A_{i}>41 \text {, and } 0 \text { otherwise. }
$$

The measures $S$ and $H$ can be viewed as independent competing risks that can be combined to form what Wilcox and Russell (1983) term the "total risk," which is, more specifically, total birth weight risk. In principle, this is also true with regard to the gestational age (preterm/postterm) measures $E$ and $L$. It follows that total risk should be modified to include the sum of all of these measures. However, risks are no longer independent, as certain combinations (early and small, heavy and late, etc.) are expected to have effects on risk beyond that predicted from main effects alone. Therefore, we include interactions between preterm/postterm risks $(E$ and $L)$ and birth weight risks ( $S$ and $H$ ) in our multivariate models.

\subsection{Modeling infant mortality}

Individual-level multivariate models of infant mortality are usually modeled as binary (lives/dies) outcomes (e.g., Hummer et al. 1999) with the log-odds of infant mortality as the usual focus of investigation. Logistic regression models of this type do not fully capture the dynamic of mortality in the first year of life. By the last decade of the 20th century, about $65 \%$ of all infant deaths in the U.S. occurred during the first month of life, with the vast majority of the latter (about 80\%) occurring during the first week (National Center for Health Statistics 1996: Table 23). For this reason, it is useful to form subdivisions by age that are more detailed than the usual dichotomy (i.e., live versus die) or trichotomy (e.g., neonatal death, postneonatal death, and survival).

Our models consider distinct periods of risk of infant death within intervals of 1st day (under 24 hours), mortality within a period of 1 day ( $\geq 24$ hours) to less than 1 
week, one week to less than 28 days, and 28 days to 1 year. We consider the following proportional hazard model for the risk of mortality $h(t)$ in the first year of life

$$
h\left(t_{i}\right)=h_{0}\left(\tau_{k}\right) \exp \left\{\mathbf{x}_{i}{ }^{\prime} \beta\left(\tau_{k}\right)\right\},
$$

where $h_{0}\left(\tau_{k}\right)$ represents a piecewise constant baseline hazard that is constant within age intervals of [0-1), [1-7), [7-28), and [28-365) days. This model allows for nonproportional covariate effects through a set of time-varying effects $\boldsymbol{\beta}\left(\tau_{k}\right)$ of covariates $\mathbf{x}$, denoting birth outcome measures and interaction terms.

Proportional hazards models with time-varying covariates have been used to study perinatal mortality using a "fetuses at risk" approach based on gestational age as the time axis (Platt et al. 2004b). This approach addresses the key issues associated with the use of gestational age as an independent variable. Specifically, the temporal nature of gestational age implies that gestational ages are not "exchangeable" in the sense that an infant born at a later age was in the risk set for all earlier birth dates, but that infants born earlier are not members of the risk sets for later gestational ages. This approach has been criticized by Wilcox and Weinberg (2004) for ignoring time since birth, arguing that both the gestational age time scale and the time since birth are relevant to an infant's risk. Accordingly, the approach we follow treats gestational age as a covariate and considers the time since birth as the temporal component of risk.

\subsection{Model specification}

We estimate the model above using the methods described by Laird and Oliver (1981), in which the data are grouped according to unique values of $S, H, E, L$, and $T$ (with $T$ denoting a factor representing the 4 age intervals). Aggregating the data this way results in a matrix of counts and exposures that can be fit using conventional log-linear models, where the number of infant deaths in each cell of the table follows a Poisson distribution and the logarithm of exposure to risk-measured in person-days in the interval-is treated as an offset term in the model. This approach yields considerable data reduction with no loss of information. We estimate the following multiplicative models applied separately to each racial and ethnic group: 


\section{Model 1:}

$$
\frac{D_{i j k l m}}{R_{i j k l m}}=\lambda_{i}^{T} \lambda_{j}^{S} \lambda_{k}^{H} \lambda_{l}^{E} \lambda_{m}^{L} \lambda_{j l}^{S E} \lambda_{k l}^{H E}
$$

and

\section{Model 2:}

$$
\frac{D_{i j k l m}}{R_{i j k l m}}=\lambda_{i}^{T} \lambda_{j}^{S} \lambda_{k}^{H} \lambda_{l}^{E} \lambda_{m}^{L} \lambda_{i j}^{T S} \lambda_{i k}^{T H} \lambda_{i l}^{T E} \lambda_{i m}^{T L} \lambda_{i j l}^{T S E} \lambda_{i j k}^{T H E},
$$

where $D_{i j k l m}$ denotes the number of infant deaths in time interval $i$, at the $j$ th value of $S$, the $k$ th value of $H$, the $l$ th value of $E$ and the $m$ th value of $L$, and $R_{i j k l m}$ is the number of person-days of exposure to risk in the ijklm cell of the $I \times J \times K \times L \times M$ table. The absence of an intercept term allows a parameterization of the baseline hazard, which contains the infant age-specific mortality rates adjusted for covariates. Certain two-way interactions such as "small $\times$ heavy" and "early $\times$ late" are not possible, thus limiting the higher-order interactions involving these terms.

Our initial model (Model 1, above) for each race/ethnic group is similar to the logit model fit by Solis et al. (2000). This model allows the baseline mortality rate to vary by age of infant but assumes age-constant (or static) effects of birth outcomes, thus providing a parsimonious baseline model for comparison with Model 2, which relaxes the assumption of age-invariant birth outcome effects. Model 1 differs from Solis et al. (2000) by estimating an age-specific baseline hazard, whereas Model 2 includes an agespecific baseline hazard in addition to age-specific birth outcome effects.

\section{Results}

\subsection{Race/ethnic variation in age-specific mortality rates}

We begin with a description of the crude age-specific infant mortality rates by race/ethnicity. Table 1 shows age-specific mortality rates for white, black, and Mexican American female infants. As might be expected, rates are lowest for each group during the postneonatal period (28-365 days). Whites and Mexican-Americans are nearly indistinguishable in terms of mortality risk during each age interval. 
Assessing the age-specific rates for these two groups, we find that the relative risk of mortality on the first day is about 9 times the risk of mortality in the first week (i.e., 939/101 for whites and 930/100 for Mexican Americans). Mortality risk during the first day is about 30 times higher than in the first month, and is more than 185 times the risk in the postneonatal period.

For blacks, the risk of mortality is about 11 times higher during the first day relative to the first week (i.e., 1794/169). This is about $14 \%$ higher than the corresponding white and Mexican-American relative risks when comparing the same two periods. The black mortality risk on the first day relative to the first month is about 30 times higher, which is similar to the corresponding relative risks among whites and Mexican-Americans. Due to the higher mortality of blacks in the postneonatal period, the relative risk of mortality on the first day is 163 times the risk in the postneonatal period, which is about $13 \%$ lower than the corresponding relative risks for the other two groups when comparing the same two periods.

Table 1:Age-specific infant mortality rates and relative risks by race/ethnicity

\begin{tabular}{|c|c|c|c|c|c|}
\hline \multirow[b]{2}{*}{ Age Interval } & \multicolumn{3}{|c|}{ Mortality Rate per $1,000,000$ Person Days ${ }^{*}$} & \multicolumn{2}{|l|}{ Relative Risk } \\
\hline & White & Black & $\begin{array}{l}\text { Mexican- } \\
\text { American }\end{array}$ & Black/White & $\begin{array}{l}\text { Mexican- } \\
\text { American/White }\end{array}$ \\
\hline day $[0-1)$ & 939 & 1794 & 930 & 1.911 & 0.991 \\
\hline day $[1-7)$ & 101 & 169 & 100 & 1.673 & 0.995 \\
\hline day $[7-28)$ & 30 & 60 & 32 & 1.976 & 1.053 \\
\hline day $[28-365)$ & 5 & 11 & 5 & 2.289 & 0.997 \\
\hline
\end{tabular}

Source: 1995-1998 NCHS linked birth/infant death files.

${ }^{*}$ Rounded to nearest whole number

The race/ethnic comparisons of mortality risk in the four age periods in Panel 2 of Table 1 confirm that there is little difference in age-specific mortality between white and Mexican-American females. The highest mortality for all groups occurs on the first day (Panel 1), with black infants facing nearly twice the risk of the other groups (Panel 2 ). Mortality risk during the first week is $67 \%$ higher for blacks compared to whites and Mexican-Americans. The mortality risk in the first month is nearly 2 times higher for blacks, whereas the risk in the postneonatal period is 2.29 times higher. Plausible explanations for the increasing relative risk with age include the possibility that blacks receive less adequate postnatal care and/or that the environment to which black infants are exposed is more hazardous than that experienced by whites and Mexican Americans (Hummer 1993). 


\subsection{Multivariate analysis}

The results in Table 1 are based on a model that includes only the baseline hazard expressed in age-specific rates per 1,000,000 person-days of life; this model reproduces the average empirical age-specific mortality rates that would be observed in the pooled 1995-1998 NCHS data. ${ }^{10}$ Next, we consider models that include the main effects of early $(E)$, small $(S)$, late $(L)$ and heavy $(H)$, in addition to the interaction terms for early $\times$ small $(E \times S)$ and early $\times$ heavy $(E \times H)$ to capture effects of prematurity and low or high birth weight occurring jointly. In all models considered here, interaction effects involving late and small $(L \times S)$ and late and heavy $(L \times H)$ were not statistically different from 0 conditional on other model effects, so we ignore these terms.

\subsubsection{Static birth outcome effects model}

Table 2 provides estimates of the baseline hazard rates in deaths per million persondays, conditional on main and interaction effects of birth outcomes for each of the two models outlined earlier. Model 1 includes only the main effects of birth outcomes and their interactions, while Model 2 allows for nonproportionality in birth outcome effects. Compared to the empirical rates shown in Table 1, the estimated baseline hazards are, as expected, greatly attenuated when controlling for birth outcomes. Model 1 accounts for 80 to 87 percent of the observed mortality, with the greatest impact on MexicanAmerican neonatal mortality and the smallest effect on black postneonatal mortality. However, despite controls for birth outcomes, the relative race/ethnic comparisons based on Model 1 of Table 2 do not differ appreciably from the race/ethnic comparisons based on the empirical age-specific rates shown in Table 1. For example, the empirical and model-based estimates of the mortality risk for backs are 1.5 to 2.5 times that of whites and Mexican-Americans for deaths occurring at all ages, with a high degree of correspondence between the empirical and model-based relative risks at any given age. Although static models of birth outcomes yield considerable reductions in predicted mortality, they do little to alter the observed age-specific patterns of relative risks. Specifically, static models do not change the overall picture of black disadvantage at any age relative to other groups.

\footnotetext{
${ }^{10}$ Letting $D_{i}$ and $R_{i}$ denote, respectively, the number of deaths and the number of person days of exposure in the ith age interval, the yearly mortality rate can be computed as $\Sigma D_{i} / R_{i}$. Letting $p_{i}$ denote the period-specific rates given in Table 1, we construct the yearly mortality rate as $\sum p_{i} R_{i}$. For example, carrying this out on the rates in Table 1 gives a yearly mortality rate for white females of 3,857 infant deaths per 1,000,000 live births per year. Note that the empirical rates will be lower in our analysis than in the national summary statistics because we have omitted plural births and infants weighing less than 500 grams at birth and less than 22 weeks gestation.
} 
Table 2: Estimated mortality and relative risks controlling for birth outcomes

\begin{tabular}{|c|c|c|c|c|c|c|}
\hline \multirow[b]{3}{*}{ Age Interval } & \multicolumn{6}{|c|}{ Model Based Estimates of Mortality Rates per $1,000,000$ Person Days } \\
\hline & \multirow{2}{*}{$\begin{array}{l}\text { White } \\
\text { Model } 1\end{array}$} & \multicolumn{3}{|c|}{ Black } & \multicolumn{2}{|c|}{ Mexican-American } \\
\hline & & Model 2 & Model 1 & Model 2 & Model 1 & Model 2 \\
\hline$[0-1)$ & 128 & 36 & 268 & 35 & 124 & 30 \\
\hline$[1-7)$ & 15 & 12 & 27 & 14 & 14 & 9 \\
\hline$[7-28)$ & 5 & 4 & 10 & 7 & 5 & 4 \\
\hline$[28-365)$ & 1 & 1 & 2 & 4 & 1 & 1 \\
\hline
\end{tabular}

\begin{tabular}{lllll}
\hline & \multicolumn{3}{l}{ Relative Risks } & \\
\cline { 2 - 5 } Age Interval & Mlack/White & & \multicolumn{2}{l}{ Mexican-American/White } \\
& Model 1 & Model 2 & Model 1 & Model 2 \\
\hline$[0-1)$ & $2.089^{*}$ & 0.962 & 0.965 & 0.954 \\
{$[1-7)$} & $1.821^{*}$ & 1.158 & 0.952 & 0.832 \\
{$[7-28)$} & $2.162^{*}$ & $1.760^{*}$ & 1.007 & 1.096 \\
{$[28-365)$} & $2.524^{*}$ & $2.673^{*}$ & 0.955 & 0.961 \\
\hline
\end{tabular}

Source: $1995-1998$ NCHS linked birth/infant death files. *Effect does not cover 1.0 with $95 \%$ confidence.

\subsubsection{Age-varying birth outcome effects model}

Model 2 of Table 2 fits varying (i.e., nonproportional) effects of birth outcomes. As might be expected due to a larger number of parameters, Model 2 produces greater attenuation in estimated mortality than Model 1, accounting for 80 to 98 percent of the observed age-specific mortality. The most dramatic reduction in expected mortality occurs for blacks during the first week of life. In fact, the estimated black/white risks are statistically equal during the first week, as indicated by estimated relative risks of 0.96 within the first day and 1.16 within first week, which do not differ statistically from 1.0. Unlike the static model, allowing for age variation in birth outcome effects accounts for the black mortality disadvantage relative to whites through the first week of life and attenuates the relative black disadvantage from the first week to the first month of life. However, neither of the models can account for the relatively higher black postneonatal mortality. One plausible explanation is that the effects of birth outcomes are strongest for causes of death that are more common during the early neonatal period (e.g., Eberstein et al. 1990). Our models in Table 2, which, due to data limitations, do not include the social and environmental conditions that are more closely 
associated with infant deaths that occur during the later infant ages, are consequently less able to explain infant mortality disparities beyond the neonatal period. This is discussed in more detail in Appendix A.

Our findings suggest that the black disadvantage during the first week of life is due to heterogeneity in the composition of birth outcomes, such that if the composition of birth outcomes were similar for blacks and whites (even considering a race-specific birth-weight standard for both groups, as in this case), differences in mortality risk during the first week would disappear. Our results are consistent with findings from other bodies of research that focus more squarely on population differences in birth outcome distributions. Researchers using parametric mixture models to identify latent primary and secondary birth weight subpopulations within advantaged (white) and disadvantaged (black) populations provide explanations for the "pediatric paradox" of lower mortality among low-birth-weight blacks compared to low-birth weight whites. Specifically, Gage et al. (2004) argue that the mortality advantage of the low-birthweight black population is due entirely to lower mortality among the low-birth-weight compromised (i.e., low birth weight, small for gestational age, intrauterine growth retarded, macrosomic, etc.) births. This research also suggests that the black compromised subpopulation may be more highly selected through fetal loss than the white compromised subpopulation.

Disparities in infant mortality are the result of differences in composition as well as differences in "return to risk." Blacks are immensely disadvantaged in compositional terms due to the much greater proportion of black births that occur at low weights and short gestations-adverse birth outcomes which are the most powerful predictors of infant death. Our findings may reflect compositional differences in the birth weight distribution. Black infants in this data set are 264 grams lighter than whites on average. The risks of low birth weight $(<2500$ grams) and very low birth weight $(<$ 1500 grams) are, respectively, 2.35 and 3.14 times higher for blacks than for whites

It is possible that racial and ethnic compositional differences in birth outcomes underlying the pediatric paradox are captured to some extent by our models, which reflect departures from gestational-age specific survival optimums and allow for differential impacts of these measures by age. For example, if deaths within the first week are disproportionately drawn from compromised subpopulations, and if the compromised subpopulation of blacks is more robust (due to greater selectivity through higher fetal loss), we should expect lower mortality risks for blacks relative to whites during the first week. Higher mortality during the later periods may reflect greater black disadvantage within the "normal" (noncompromised) subpopulation. 


\subsubsection{Description of birth outcome effects}

We turn now to a direct examination of birth outcome effects from Models 1 and 2 . Table 3 compares the age-invariant birth outcome effects of Model 1 to the age-varying effects of Model 2, thus providing a direct assessment of variation in birth outcome effects and a comparison to the corresponding age-invariant effects. The second panel of Table 3 displays ratios of estimated birth outcome effects for blacks and Mexican Americans relative to the corresponding estimates for whites. In addition to discussing the estimates presented in Table 3, we will also make comparisons of effects within this table in order to assess differences in these effects by age. It should be noted that all main effects (early, late, small, and heavy) showed evidence of age variation when age periods [0-1) , [1-7), and [7-28) are contrasted against postneonatal baseline effects.

We focus first on birth weight outcomes "small" and "heavy." Table 3 shows that being smaller or heavier than the gestational age-specific optimum has the largest effect on infant mortality for all three race/ethnic groups. The effects of being more than one standard deviation below the gestational age-specific optimal birth weight diminish with age, and are generally larger for whites and Mexican-Americans and smallest among blacks. However, we find significant race/ethnic differences only when comparing black/white mortality beyond the first week. For example, working directly with the age-specific effects, we note that the effects of small birth weight on mortality occurring in the neonatal period are uniformly higher (15 to 86 percent higher per standard deviation below the gestational age specific optimum) than in the postneonatal period. Among blacks, as compared to Mexican Americans and whites, the risk associated with low birth weight is uniformly smaller in both Models 1 and 2-a result consistent with the previously documented survival advantage of low birth weight blacks, compared to their white counterparts (Kline, Stein, and Susser 1989; Wilcox and Russell 1986, 1990). With regard to age-variation in the low birth weight effect for blacks relative to other groups, we find that the risk associated with low birth weight for blacks in the first day is $18 \%$ higher than in the postneonatal period. ${ }^{11}$

The effect of being heavier than the optimum weight for gestational age also varies by age (Table 3, Panel 1). If we ignore age variation (Model 1), the effect of being more than one standard deviation above the gestational age-specific mean birth weight is to increase mortality by $63 \%$ to $108 \%$ (depending on race/ethnicity) per standard deviation increase in birth weight beyond the optimum, with the smallest effect observed for blacks and the largest effect for Mexican-Americans. We find a pattern of monotonic decline in the effect of heavy birth weight by age for whites and blacks, whereas the age

${ }^{11}$ All the relative comparisons in age-varying effects we discuss are significantly different from 1.0. 
Table 3: Fixed and varying effects of birth outcomes on infant mortality: U.S. females, 1995-1998

\begin{tabular}{|c|c|c|c|c|c|c|c|c|c|c|}
\hline \multirow{3}{*}{ Multiplicative Effects } & \multirow{2}{*}{\multicolumn{2}{|c|}{ White }} & \multirow{2}{*}{\multicolumn{2}{|c|}{ Black }} & \multirow{2}{*}{\multicolumn{2}{|c|}{ Mexican -American }} & \multicolumn{4}{|c|}{ Race/Ethnic Comparisons } \\
\hline & & & & & & & Black/Wr & & $\begin{array}{l}\text { Mexican- } \\
\text { American }\end{array}$ & White \\
\hline & Model 1 & Model 2 & Model 1 & Model 2 & Model 1 & Model 2 & Model 1 & Model 2 & Model 1 & Model 2 \\
\hline early & $1.407^{*}$ & --- & $1.274^{*}$ & --- & $1.356^{*}$ & --- & $0.906^{*}$ & --- & $0.964^{*}$ & --- \\
\hline early $[0-1)$ & & $1.577^{*}$ & & $1.484^{*}$ & & $1.479^{*}$ & & $0.941^{*}$ & & $0.938^{*}$ \\
\hline early $[1-7)$ & & $1.436^{*}$ & & $1.324^{*}$ & & $1.388^{*}$ & & $0.922^{*}$ & & 0.967 \\
\hline early [7 - 28) & & $1.415^{*}$ & & $1.320^{*}$ & & $1.377^{*}$ & & $0.933^{*}$ & & 0.973 \\
\hline early [28 - 365) & & $1.264^{*}$ & & $1.191^{*}$ & & $1.262^{*}$ & & $0.943^{*}$ & & 0.999 \\
\hline late & $1.141^{*}$ & --- & $1.179^{*}$ & --- & $1.145^{\star}$ & --- & 1.034 & --- & 1.003 & --- \\
\hline late $[0-1)$ & & $1.102^{*}$ & & $1.307^{*}$ & & $1.213^{*}$ & & $1.186^{*}$ & & 1.101 \\
\hline late $[1-7)$ & & 1.044 & & $1.185^{*}$ & & 1.081 & & 1.135 & & 1.035 \\
\hline late $[7-28)$ & & $1.142^{*}$ & & $1.208^{*}$ & & 1.053 & & 1.057 & & 0.922 \\
\hline late [28-365) & & $1.127^{*}$ & & $1.106^{*}$ & & $1.130^{*}$ & & 0.981 & & 1.002 \\
\hline small & $2.266^{*}$ & --- & $1.813^{*}$ & --- & $2.253^{*}$ & --- & $0.800^{*}$ & --- & 0.994 & \\
\hline small $[0-1)$ & & $3.123^{*}$ & & $3.022^{*}$ & & $3.174^{*}$ & & 0.968 & & 1.016 \\
\hline small [1 - 7) & & $2.484^{*}$ & & $2.240^{*}$ & & $2.640^{*}$ & & 0.902 & & 1.063 \\
\hline small [7 - 28) & & $2.365^{*}$ & & $1.951^{*}$ & & $2.228^{*}$ & & $0.825^{*}$ & & 0.942 \\
\hline small [28 - 365) & & $1.976^{*}$ & & $1.626^{*}$ & & $1.933^{*}$ & & $0.823^{*}$ & & 0.979 \\
\hline heavy & $1.842^{*}$ & -- & $1.627^{*}$ & --- & $2.077^{*}$ & --- & $0.883^{*}$ & --- & 1.127 & \\
\hline heavy $[0-1)$ & & $2.520^{*}$ & & $2.615^{\star}$ & & $1.881^{*}$ & & 1.038 & & 0.747 \\
\hline heavy $[1-7)$ & & $2.208^{*}$ & & $2.127^{\star}$ & & $2.820^{*}$ & & 0.963 & & 1.277 \\
\hline heavy [7 - 28) & & $2.040^{*}$ & & $1.821^{*}$ & & $1.988^{*}$ & & 0.893 & & 0.975 \\
\hline heavy [28 - 365) & & $1.434^{*}$ & & $1.254^{\star}$ & & $1.789^{*}$ & & 0.874 & & $1.247^{*}$ \\
\hline early $\times$ small & $1.030^{*}$ & --- & $1.055^{\star}$ & --- & $1.043^{*}$ & --- & $1.025^{\star}$ & --- & $1.013^{*}$ & --- \\
\hline early $\times$ small $[0-1)$ & & $1.018^{*}$ & & $1.037^{\star}$ & & $1.046^{*}$ & & $1.019^{*}$ & & $1.027^{*}$ \\
\hline early $\times$ small $[1-7)$ & & $1.027^{*}$ & & $1.061^{*}$ & & $1.039^{*}$ & & $1.032^{*}$ & & 1.011 \\
\hline early $\times$ small $[7-28)$ & & $1.036^{*}$ & & $1.054^{\star}$ & & $1.046^{*}$ & & 1.017 & & 1.009 \\
\hline early $\times$ small $[28-365$ ) & & $1.025^{*}$ & & $1.035^{\star}$ & & $1.026^{*}$ & & 1.010 & & 1.001 \\
\hline early $\times$ heavy & $0.935^{*}$ & --- & $0.957^{*}$ & --- & $0.957^{*}$ & --- & $1.023^{*}$ & --- & 1.024 & --- \\
\hline early $\times$ heavy $[0-1)$ & & $0.949^{*}$ & & $0.960^{*}$ & & 0.996 & & 1.012 & & 1.049 \\
\hline early × heavy $[1-7)$ & & $0.908^{*}$ & & $0.918^{*}$ & & $0.882^{*}$ & & 1.011 & & 0.971 \\
\hline early × heavy [7 - 28) & & $0.904^{*}$ & & $0.939^{*}$ & & 0.956 & & 1.039 & & 1.057 \\
\hline early $\times$ heavy $[28-365$ ) & & $0.927^{*}$ & & $0.960^{*}$ & & 0.969 & & 1.036 & & 1.045 \\
\hline
\end{tabular}

Source : 1995-1998 NCHS linked birth/infant death files. *Effect does not cover 1.0 with 95\% confidence. 
pattern for Mexican-Americans is generally inverted J-shaped. Moreover, for blacks and whites, there are significant differences in the heavy birth weight effects when comparing effects in the neonatal period to those in the postneonatal period. For example, the effect on mortality within the first day is 1.76 to 2.09 times higher-per standard deviation increase in birth weight beyond the gestational age-specific optimum-relative to its effect on mortality in the postneonatal period for whites and blacks respectively. For Mexican-Americans, there is evidence of a larger relative effect of heavy birth weight on mortality, but only during the first week. The only noticeable racial/ethnic difference in the effect of heavy birth weight occurs between Mexican-Americans and whites, with a standard deviation increase in birth weight above the gestational age-specific optimum increasing the postneonatal mortality risk of Mexican-Americans by $25 \%$ over that of whites.

We now discuss the effects of gestational age. Table 3 shows that gestational age earlier than the 39th week has a moderate impact on all race/ethnic groups, with a somewhat larger effect for whites and Mexican-Americans than for blacks. These effects diminish with age for all groups, such that the effects of early gestational age on neonatal mortality across all groups are 10 to 25 percent higher (per week of gestational age below the 39th week) than the corresponding effects on postneonatal mortality, with larger neonatal/postneonatal differences evident for whites and blacks. Panel 2 of Table 3 shows that the effect of early gestational age among blacks is 92 to 94 percent of the white effect. The impact of short gestation among Mexican-Americans is somewhat smaller compared to whites within the first day, but there is no evidence of Mexican-American/white differences for the other age intervals (based on a statistical comparison of the relative effects across selected age intervals).

Thus, while race/ethnic differences in the effect of early gestational age are apparent (and statistically different from 1.0), these differentials seem small in comparison to those of birth weight. However, it is important to note that gestational age measures refer to a difference of one week in gestational age, and it is therefore much more likely to find such a difference than a difference of one standard deviation in birth weight. Therefore, the apparently small black/white differential is likely a result of the scale of measurement of "early" and "late" outcomes, which is not comparable to the scale of measurement of "small" and "heavy" outcomes. As such, the gaps of $6 \%$ to $8 \%$ in the effect of "early" gestational age are very significant given the larger observed variations in early gestational age in relation to birth weight. In this respect, the finding of a less harmful effect of early delivery among blacks may be as important as the paradoxical survival advantage of low birth-weight black infants noted by Wilcox and Russell (1990) among others. This finding of a relative survival advantage of premature black infants (net of birth weight) has been noted by other authors (Adams et al. 1991; Allen et al. 2000; Alexander et al. 2003). 
Blacks face the greatest mortality disadvantage relating to gestational age later than the 41 st week. The effects at all ages are statistically different from 1.0, with the largest effect (1.307) occurring on the first day. Among Mexican-Americans, the effects of late birth are generally smaller and significantly different from 1.0 only within the first day and during the postneonatal period. Among whites, age-varying effects of late birth are significant (except for days 1 to 7 ), and roughly similar in pattern to the results for blacks. Race/ethnic comparisons in Panel 2 show that the risks (relative to whites) associated with late birth are small for blacks and Mexican Americans, with the only significant effect being that blacks face a risk that is $18 \%$ higher than whites within the first day of life. In terms of age-variation, the risk of late gestational age on mortality in the first day-relative to postneonatal mortality-is $21 \%$ higher (per week beyond the 41st) for blacks compared to whites or MexicanAmericans. Accounting for age variation in the effect of late gestational age (especially on mortality within the first day) may contribute to the elimination of the observed black disadvantage (relative to whites) in the first week of life as shown in Table 2.

Being born both early and small is associated with a modest additional increase in risk, whereas being born early and heavy additionally lowers the risk for all groups beyond the main effects. The third-order interactions allowing for age-varying effects are "offsetting" in the sense that the early $\times$ small $\times$ age interaction increases risk slightly, and the early $\times$ heavy $\times$ age term reduces risk. Results show no evidence of a monotonic pattern of decrease or increase in either of these effects by age, and the effects of these interactions on neonatal mortality do not differ significantly from their corresponding effects on postneonatal mortality. Therefore, simple interaction termsrather than age-varying interactions-would yield a more parsimonious model.

The main relationships described above may be summarized graphically as in Figures 1 and 2, which show the dynamics of the major birth outcome effects by age in a comparison of whites and blacks. Figure 1 shows the multiplicative effects associated with a one standard deviation decrease below the gestational age- and racespecific optimum birth weight, and the behavior of this effect during specific age periods during the first year. The effects for whites are respectively 3.123, 2.484, 2.365, and 1.976 times higher for each standard deviation below optimum birth weight in each of the age intervals. The effects for blacks are somewhat lower than the white effects in the first two age intervals (3.022 and 2.240), but are markedly lower in the later two periods (1.951 and 1.626). These later effects are about $17 \%$ lower than the corresponding effects for whites. Both groups exhibit significant differences between the earlier effects and the effects during the postneonatal period.

Figure 2 provides a similar comparison of "early" gestational age (effect per week below the 39th). These effects show the same pattern of attenuation over time, with a smaller impact on the black mortality risk (between 92 and 94 percent of the white 
effect). However, given the low variability in gestational age, these apparently small effects (measured in weeks) represent significant multipliers of the mortality risk. This graphical evidence suggests that simpler models of linear age variation in the birth outcome effect may be adequate.

Figure 1: $\quad$ Race-specific and relative effects of being more than one standard deviation below the race-specific optimum birth weight for whites and blacks by age-interval.

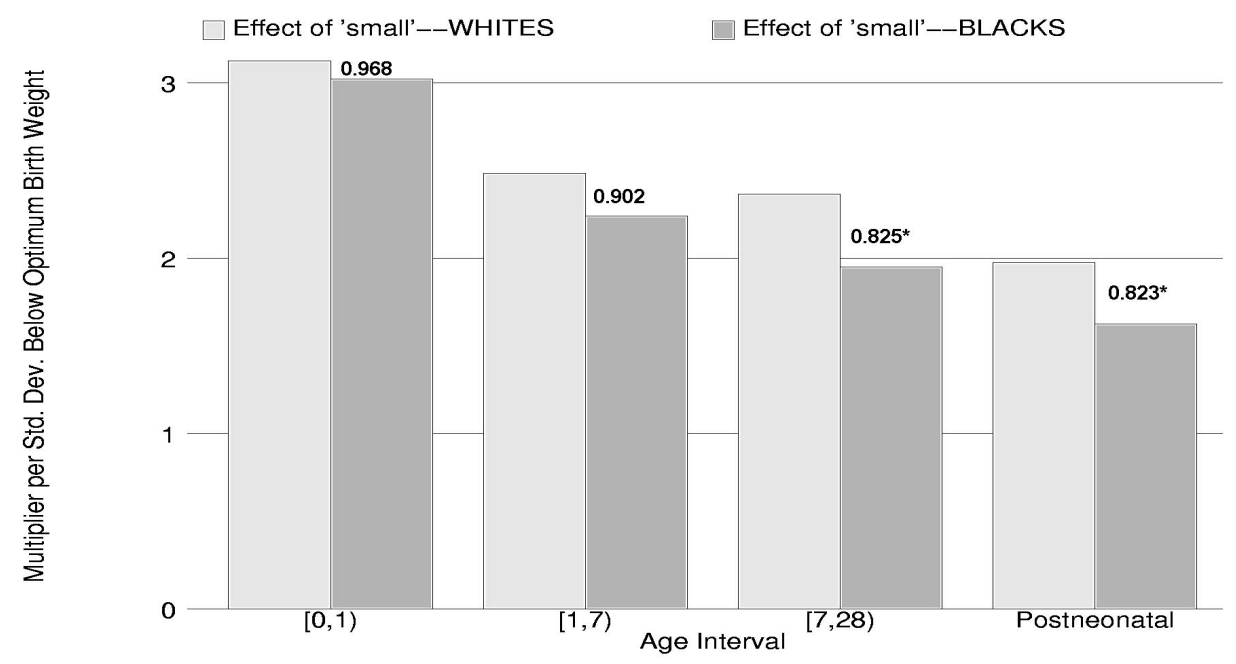


Figure 2: $\quad$ Race-specific and relative effects of being more than one week below the 39th week of gestational age for whites and blacks by age-interval.

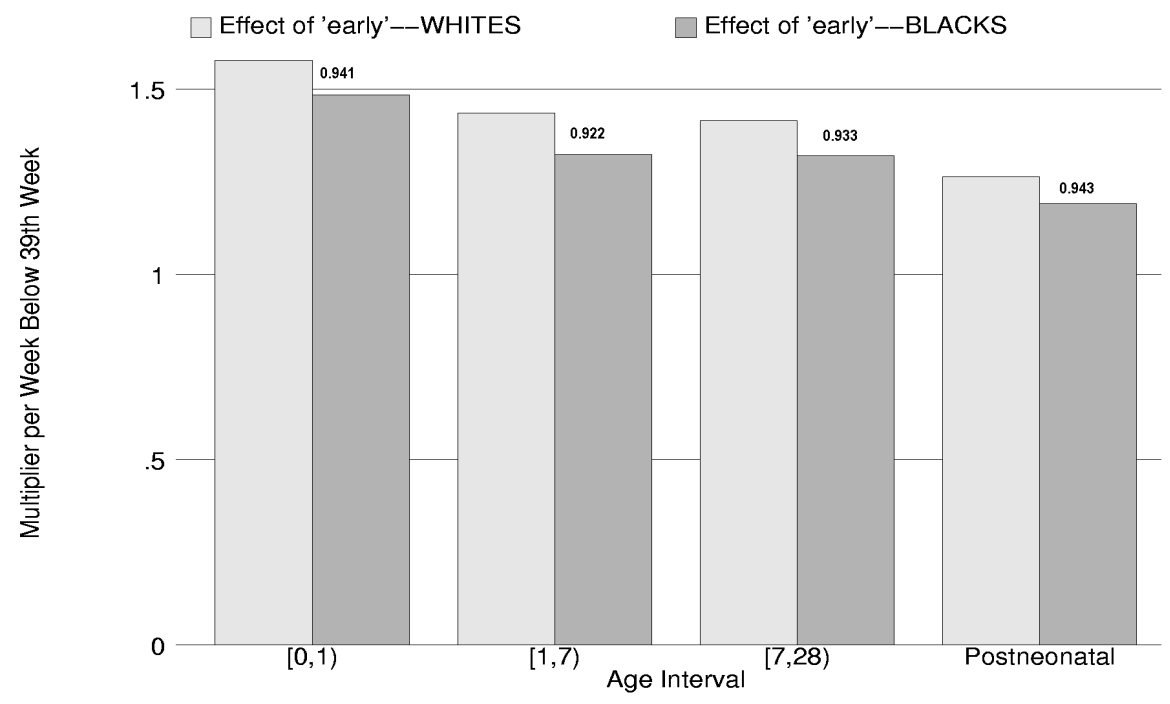

\section{Discussion}

This paper investigates infant mortality in the U.S. for the years 1995-1998 for white, black, and Mexican-American females. The results of a relatively straightforward expansion of the work of Solis et al. (2000) to include blacks and Mexican Americans showed that the risk of infant death in each of the race/ethnic groups notably increased at birth weights lower or higher than the optimum and early gestational age. In the case of both blacks and Mexican Americans, just as for whites, being born both early and light resulted in a modestly and significantly higher risk than would be expected based on main effects. Conversely, infants born at weights that are heavy relative to gestational age were at reduced risk compared to what would be expected based on main effects only.

Importantly, the relationships just summarized show evidence of nonproportional effects, with a tendency for larger effects on neonatal mortality and diminishing effects at older infant ages. Age varying effects of birth outcomes account for the relatively 
higher mortality experienced by black females in the early neonatal period (from the 1 st to the 7 th days), and to some degree during the later neonatal period ( $7^{\text {th }}$ to $28^{\text {th }}$ days). Our tentative conclusion based on a series of simpler models described in Appendix A is that age-varying effects of early and late gestational age and small birth weight statistically account for the observed black disadvantage in the early neonatal period (less than one week). Lack of variation in the effects of late gestational age for whites and Mexican-Americans may be partially responsible for this finding. Specifically, the effect of late gestational age on mortality in the first day relative to the postneonatal mortality is about $21 \%$ higher for blacks than the corresponding age effect for whites and Mexican-Americans. Our models are less able to account for the relatively higher mortality experienced by black females in the late neonatal (one week to one month) and postneonatal periods, periods of time when social and environmental influences on differential mortality risk begin to become more pronounced together with a greater likelihood of infants dying of more exogenous, rather than endogenous, causes (Eberstein et al. 1990). ${ }^{12}$

The reduced black/white differential in neonatal mortality risk may also be the result of a relatively larger effect of small birth weight on mortality in the first day relative to postneonatal mortality, which is about $18 \%$ higher in the black population than the corresponding white effect. Disparities in infant mortality are the result of differences in composition as well as differences in "return to risk." Blacks are immensely disadvantaged in compositional terms due to the much greater proportion of black births that occur at low weights and short gestations-adverse birth outcomes that are the most powerful predictors of infant death. Our findings may reflect compositional differences in the birth weight distribution.

A clear limitation of the present work is the assumption that one standard deviation above the gestational age-specific mean birth weight defines the survival optimum birth weight for all racial and ethnic groups. Although one standard deviation appears to represent a reasonably close approximation of the survival optimum for white female infants, deviations of greater or lesser magnitude may more closely reflect the optimum for other race/ethnic groups. Our research uses race/ethnic-specific measures of birth weight to construct gestational age-specific scores that denote departure from average birth weight for a particular gestational age. But this constitutes only a partial response to the concern raised by Alexander et al. (1999b) about the validity of the "one-sizefits-all" assumption. Specifically, these authors suggest that because "the general norms for preterm, postterm, and fetal growth measures may be largely derived from white populations, more information is needed to assess whether or not the ongoing use of these one-size-fits-all standards may result, for other ethnic groups, in invalid risk

\footnotetext{
12 Exogenous causes of deaths are generally thought of as relating more to environmental and external factors, whereas endogenous causes of death are usually attributed to genetic characteristics of the infant, characteristics of life in utero, and the conditions of labor (Hummer 1993).
} 
assessments and the misidentification of infants in need of intervention services" (Alexander et al. 1999b: 77). To fully address this issue, it would be necessary to determine as precisely as possible the magnitude of the deviation from a race/ethnicspecific mean that is required to maximize survival for that specific group. A promising area of future research will be to rigorously determine race/ethnic-specific optima, a process begun by Echeverria-Cruz (2004) and Adams et al. (1991). Wilcox and Russell (1986) show how this can be done using birth weight as a covariate in two separate logit models that partition the perinatal mortality risk into two separate risks, one that decreases with birth weight and one that increases. The optimum is determined by the intersection of the two fitted logit lines. However, this approach does not consider gestational age-specific optima. Adams et al. (1991) provide an approach based on fitting logit models for perinatal mortality with linear and quadratic birth weight terms to 4 gestational age intervals. Both Adams et al. (1991) and Wilcox and Russell (1986) find that birth weight optimums for perinatal mortality are closer to the mean (or median) birth weight for blacks.

We have taken steps that expand on the work by Adams et al. (1991). Appendix A of this paper applies a modeling approach to determine the survival optimum for each racial and ethnic group we consider. However, in the present context we consider females only and do not limit the focus to perinatal mortality. We find that the survival optima for whites and blacks in the 37-42 week gestational age race are very close to one standard deviation above the respective gestational age-specific means, whereas for Mexican Americans, the survival optimum is located about $2 / 3 \mathrm{rds}$ of a standard deviation above the mean. It seems unlikely that these differences are large enough to change the central findings presented here with respect to patterns of age variation in birth outcome effects (and their differences) for whites and blacks. Further examination of these effects for Mexican Americans is needed.

Another limitation related to the use of a gestational age birth weight standard is the assumption that the gestational age-specific birth weight optimum is constant over the first year. The empirical evidence presented in Appendix A suggests that the optima are further from the mean for preterm births and closer to the mean for later term births. This however, does not address the possibility that the optimum shifts from one piece of the proportional hazards model to another. For example, high mortality for macrosomic infants in the neonatal period may select out heavier infants so that the postneonatal optimum birth weight optimum is shifted toward smaller birth weights.

We mentioned that there is considerable debate on the use of population-specific standards in the study of infant mortality. Whether the standardizations used here introduce bias into race/ethnic comparisons must await further investigation. New approaches using percentile-based methods of standardization may offer additional insights (Hertz-Picciotto and Din-Dzietham 1998). To the extent that the 
standardization we use leads to findings that are consistent with the general conclusions reached using cutpoint methods, but provide greater detail on birth outcome dynamics in the first year of life, there is much to recommend this approach. Of greatest interest, however, is in understanding the biological mechanisms that link birth outcomes to mortality, and the role of social environment on these mechanisms. Further insight into these issues will likely come from specialists working with data that can more squarely address the biological and social-environmental foundations of birth outcomes.

\section{Acknowledgements}

The authors gratefully acknowledge the support for this analysis provided by the National Institute of Child Health and Human Development under grant No. R01 HD41147. We would like to thank members of the mortality group at the Population Research Center at the University of Texas at Austin and the two reviewers for helpful comments on an earlier version of this paper. 


\section{References}

Adams, N.M., C.J. Berg, P.H. Rhodes, and B.J. McCarthy. 1991. "Another Look at the Black-White Gap in Gestation-Specific Perinatal Mortality." International Journal of Epidemiology, 20: 950-957.

Albrecht, S.L., L.L. Clarke, M.K. Miller, and F.L. Farmer. 1996. "Predictors of Differential Birth Outcomes among Hispanic Subgroups in the United States: The Role of Maternal Risk Characteristics and Medical Care." Social Science Quarterly 77: 407-433.

Allen, M.C., G.R. Alexander, M.E. Tompkins, and T.C. Hulsey, 2000 "Race Differences in Temporal Changes in Newborn Viability and Survival by Gestational Age." Paediatric and Perinatal Epidemiology, 14: 152-158.

Alexander, G.R., M.D. Kogan, and J.H. Himes. 1999a. "1994-1996 U.S. Singleton Birth Weight Percentiles for Age by Race, Hispanic Origin, and Gender." Maternal and Child Health Journal 3: 225-232.

Alexander, G.R., M.E. Tompkins, M.C. Allen, and T.C. Hulsey. 1999b "Trends and Racial Differences in Birth Weight and Related Survival." Maternal and Child Health Journal 3: 71-79.

Alexander, G R., M.D. Kogan, D. Bader, W. Carlo, M.C. Allen, and J. Mor. 2003 "US Birth Weight/Gestational Age-Specific Neonatal Mortality: 1995-1997 Rates for White, Hispanics, and Blacks." Pediatrics, 111: 61-66.

Battaglia, F.C., and L.O. Lubchenco. 1967. "A Practical Classification of Newborn Infants by Weight and Gestational Age." Journal of Pediatrics 71: 159-163.

Becerra, J.E., C.J.R. Hogue, H. Atrash, and N. Pérez. 1991. "Infant mortality among Hispanics: A Portrait of Heterogeneity ." Journal of the American Medical Association 265:217-221.

Buehler, J.W., J.C. Kleinman, C.J. Hogue, L.T. Strauss, and J.C. Smith. 1987. "Birth Weight-Specific Infant Mortality, United States, 1960-1970.” Public Health Reports 102: 151-161.

Chung, C.S. 1967. "Birthweight," Pp. 92, In Morton, N.E, C.S. Chung, and M. P. Mi, Eds., Genetics of Interracial Crosses in Hawaii, Basel: Karger.

Cobas, J.A., H. Balcazar, M. Benin, V.M. Keith, and Y. Chong. 1996. "Acculturation and Low-Birthweight Infants among Latino Women: A Reanalysis of HHANES 
Data with Structural Equation Models." American Journal of Public Health 86: 394-396.

Cramer, J.C. 1987. "Social Factors and Infant Mortality: Identifying High-Risk Groups and Proximate Causes." Demography 24: 299-322.

David, R. 2001. "Commentary: Birthweights and Bell Curves." International Journal of Epidmiology 30: 1241-1243.

Echeverria-Cruz, S. 2004. "U.S. Models of Infant Mortality by Race/Ethnicity: One Size Fits All? Paper Presented at the Annual Meeting of the Population Association of America, Boston, MA.

Eberstein, I.W., C.B. Nam, and R.A. Hummer. 1990. "Infant Mortality by Cause of Death: Main and Interaction Effects." Demography 27: 413-430.

Frisbie, W.P. 2005. "Infant Mortality." pp. 251-282 in D.L. Poston, Jr., and M. Micklin (eds.) Handbook of Population. New York: Kluwer/Plenum/Springer.

Frisbie, W.P., D. Forbes, and S.G. Pullum. 1996. "Compromised Birth Outcomes and Infant Mortality among Racial and Ethnic Groups.” Demography 33: 469-481.

Frisbie, W.P., M. Biegler, P.B. de Turk, D. Forbes, and S.G. Pullum. 1997. "Racial and Ethnic Differences in Determinants of Intrauterine Growth Retardation and Other Compromised Birth Outcomes." American Journal of Public Health 87: 1977-1983.

Frisbie, W.P., D. Forbes, and R.A. Hummer. 1998. "Hispanic Pregnancy Outcomes: Additional Evidence." Social Science Quarterly 79: 149-169.

Frisbie, W.P., and S.E. Song. 2003. "Hispanic Pregnancy Outcomes: Differentials over Time and Current Risk Factor Effects." Policy Studies Journal 31: 237-252.

Gage, T.B., M.J. Bauer, N. Heffner, and H. Stratton. 2004. "Pediatric Paradox: Heterogeneity in the Birth Cohort." Human Biology 76: 327-342.

Gortmaker, S.L., and P.H. Wise. 1997. "The First Injustice: Socioeconomic Disparities, Health Services Technology, and Infant Mortality." Annual Review of Sociology 23: $147-170$.

Guendelman, S., and B. Abrams. 1995. "Dietary Intake among Mexican-American Women: Generational Differences and a Comparison with White Non-Hispanic Women" American Journal of Public Health 85: 20-25.

Guyer, B., M.F. MacDorman, J.A. Martin, K.D. Peters, and D.M. Strobino. 1998. “Annual Summary of Vital Statistics-1997." Pediatrics 102: 1333-1349. 
Hack, M., N.K. Klein, and H.G. Taylor. 1995. "Long-Term Developmental Outcomes of Low Birth Weight Infants" The Future of Children 5: 176-196.)

Hertz-Picciotto, I. 2001. "Commentary: When Brilliant Insights Lead Astray." International Journal of Epidemiology 30: 1243-1244.

Hertz-Picciotto, I, and R. Din-Dzietham. 1998. "Comparisons of Infant Mortality Using a Percentile-Based Method of Standardization for Birthweight or Gestational Age. Epidemiology 1:67-68.

Hoyert, D.L., E. Arias, B.L. Smith, S.L. Murphy, and K.D. Kochanek. 2001. "Deaths: Final Data for 1999." National Vital Statistics Reports 49, No. 8. Hyattsville, MD: National Center for Health Statistics.

Hummer, R.A. 1993. "Racial Differentials in Infant Mortality in the U.S.: An Examination of Social and Health Determinants." Social Forces 72: 529-554.

Hummer, R.A. 1996. "Black-White Differences in Health and Mortality." The Sociological Quarterly 37: 105-125.

Hummer, R.A., C.P. Schmertmann, I.W. Eberstein, and S. Kelly. 1995. "Retrospective Reports of Pregnancy Wantedness and Birth Outcomes in the United States." Social Science Quarterly 76: 402-418.

Hummer, R.A., M. Biegler, P.B. de Turk, D. Forbes, W.P. Frisbie, Y. Hong, and S.G. Pullum. 1999. "Race/Ethnicity, Nativity, and Infant Mortality in the United States." Social Forces 77: 1083-1118.

Kallan, J.E. 1993. "Race, Intervening Variables, and Two Components of Low Birth Weight." Demography 30: 489-506.

Kiely, J., and M. Susser. 1992. "Preterm Birth, Intrauterine Growth Retardation, and Perinatal Mortality.” American Journal of Public Health 82: 343-345.

Kline, J., Z. Stein, and M. Susser. 1989. Conception to Birth: Epidemiology of Prenatal Development. New York: Oxford.

Kramer, M.S., F.H. McLean, M.E. Boyd, and R.H. Usher. 1988. "The Validity of Gestational Age Estimation by Menstrual Dating in Term, Preterm, and Postterm Gestations." Journal of the American Medical Association 260: 3306-3308.

Kramer, M.S., F.H. McLean, M. Olivier, D.M. Willis, and R.H. Usher. 1989. "Body Proportionality and Head and Length 'Sparing' in Growth-Retarded Neonates: A Critical Reappraisal.” Pediatrics 84: 717-723. 
Laird, N., and D. Olivier. 1981. "Covariance Analysis of Censored Survival Data Using Log-linear Analysis Techniques" Journal of the American Statistical Association 76: 231-240.

Mathews, T.J., F. Menacker, and M.F. MacDorman. 2002. "Infant Mortality Statistics from the 2000 Period Linked Birth/Infant Death Data Set." National Vital Statistics Reports 50(12). Hyattsville, MD: National Center for Health Statistics.

McCormick, M.C. 1985. "The Contribution of Low Birth Weight to Infant Mortality and Childhood Mortality." New England Journal of Medicine 312: 82-90.

Minono, A.M., E. Arias, K.D. Kochanek, S.L. Murphy, and B.L. Smith. 2002. "Deaths: Final Report Data for 2000." National Vital Statistics Reports 50(15). Hyattsville, MD: National Center for Health Statistics.

Myers, S.A., and R. Ferguson. 1989. "A Population Study of the Relationship between Fetal Death and Altered Fetal Growth." Obstetrics and Gynecology 74: 325-331.

National Center for Health Statistics. 1996. Health, United States, 1995. Hyattsville, MD: Public Health Service.

Palloni, A., and J.D. Morenoff. 2001. "Interpreting the Paradoxical in the Hispanic Paradox." Annals of the New York Academy of Sciences 954: 140-174.

Platt, R.W., C.V. Ananth, and M.S. Kramer. (2004a) "Analysis of Neonatal Mortality: Is Standardizing for Relative Birth Weight Biased?" BMC Pregnancy and Childbirth. 4: 9.

Platt, R.W., K.S. Joseph, C.C. Ananth, J. Gondines, M. Abramowicz, and M. S. Kramer. (2004b). "A Proportional Hazards Model with Time-Dependent Covariates and Time-Varying Effects for Analysis of Fetal and Infant Death." American Journal of Epidemiology, 160: 199-206.

Preston, S.H., P. Heuveline, and M. Guillot. 2001. Demography: Measuring and Modeling Population Processes. Oxford, UK: Blackwell Publishers.

Rumbaut, R., and J.R. Weeks. 1991. "Perinatal Risks and Outcomes among Low Income Immigrant." Final Report for the Maternal and Child Health Research Program. Rockville: DHHS.

Rumbaut, R.G., and J.R. Weeks. 1996. "Unraveling a Public Health Enigma: Why Do Immigrants Experience Superior Perinatal Health Outcomes?" Research in the Sociology of Health Care 13b: 337-391. 
Scribner, R. 1996. "Editorial: Paradox as Paradigm - The Health Outcomes of Mexican Americans." American Journal of Public Health 86: 303-305.

Scribner, R., and J.H. Dwyer. 1989. "Acculturation and Low Birth Weight among Latinos in the Hispanic HANES" American Journal of Public Health 79: 12631267.

Solis, P., S.G. Pullum, and W.P. Frisbie. 2000. "Demographic Models of Birth Outcomes and Infant Morality: An Alternative Measurement Approach." Demography 37: 489-498.

U.S. Department of Health and Human Services. 1995. "Linked Birth/Infant Death Data Set: 1989 Cohort." Public Use Data File Documentation. Hyattsville: National Center for Health Statistics.

Wilcox, A.J. 2001a. "On the Importance - and the Unimportance - of Birthweight." International Journal of Epidemiology 30: 1233-1241.

Wilcox, A.J. 2001b. "Response: Where Do We Go from Here." International Journal of Epidemiology 30:1245.

Wilcox, A.J. 2003. "Commentary: On the Paradoxes of Birthweight." International Journal of Epidemiology 32: 632-633.

Wilcox, A.J., and I.T. Russell. 1983. "Birthweight and Perinatal Mortality: II. On Weight-Specific Mortality.” International Journal of Epidemiology 12: 319-325.

Wilcox, A.J., and I.T. Russell. 1986. "Birthweight and Perinatal Mortality: III. Towards a New Method of Analysis." International Journal of Epidemiology 15: 188196.

Wilcox, A.J., and I.T. Russell. 1990. "Why Small Black Infants Have a Lower Mortality Rate than Small White Infants: The Case for Population-Specific Standards for Birth Weight." Journal of Pediatrics 116: 7-10.

Wilcox, A.J., and R. Skjœrven. 1992. "Birth Weight and Perinatal Mortality: The Effect of Gestational Age." American Journal of Public Health 82: 378-382

Wilcox, A.J., and C. R. Weinberg. 2004. "Invited Commentary: Analysis of Gestational-Age-Specific Mortality - On What Biologic Foundations?" American Journal of Epidemiology 160:213-214.

Wise, P.H. 2003. "The Anatomy of a Disparity in Infant Mortality." Annual Review of Public Health 24: 341-362. 
Powers et al.: Race/Ethnic differences and age-variation

Wise, P.H., and D. Pursely. 1992. "Infant Mortality as a Social Mirror." New England Journal of Medicine 326: 1558-1560.

Yerushalmy, J. 1967. "The Classification of Newborn Infants by Birth Weight and Gestational Age." Journal of Pediatrics 71: 164-172. 


\section{Appendix A}

\section{Race/Ethnic specific birth weight optima}

In this section we describe a simple method to estimate race/ethnic specific gestational age-specific survival optimal birth weight from individual-level data. Solis et al. (2000) determined the survival optimum for white females in the 1991-1995 linked files by first standardizing the birth weight distribution around the mean birth weight in the 3941 week gestational age range. This aggregation of data yielded sufficient observations to compute the empirical IMRs for different $z$-score standardized birth weights. They then examined log IMR over a grouping of $z$-values. The survival optimum (i.e., the birth weight yielding the lowest log IMR) was found to be between 1.00-1.99 standard deviations above the mean birth weight for gestational ages in this range. Thus, the value of 1 standard deviation above the gestational age specific mean birth weight was chosen a lower bound on the survival optimum. This value was then applied to construct the birth weight risk measures $S$ and $H$ for all infants over the 22-47 week gestational age range.

Our method follows a similar logic, but is based on fitting a statistical model for mortality (on the individual-level data) as a function of gestational age-standardized birth weight. We first construct a standard score, $Z$, representing an infant's departure from her gestational age-specific mean birth weight in standard deviation units. We then determine the survival optimal birth weight for gestational ages in the 37-42 week range (i.e., for between $80 \%$ to $88 \%$ of births, depending on race/ethnicity), and for gestations $<37$ weeks and $>42$ weeks.

Methods to determine race-specific optima must account for the inverted J-shaped relationship between gestational-age standardized birth weight and mortality. To account for this nonlinearity, we estimate logit models with polynomial terms in $Z$. Similar approaches to determining race specific optima for perinatal mortality have been used by Adams et al. (1991). This simple parametric strategy has the advantage of producing point estimates and standard errors that may be useful in future work to compute upper and lower bounds on survival optima. The results presented below are based on fitting 5th-degree polynomials. Letting $p_{i}$ denote the probability of mortality for the $i$ th infant, the log odds of mortality can be expressed as a $K$ th-degree polynomial in $Z$ as

$$
\eta_{i}=\operatorname{logit}\left(p_{i}\right)=\beta_{0}+\sum_{k=1}^{K} \beta_{k} Z_{i}^{k}
$$


The estimated survival optimum is a point on the $Z$ scale that is the solution to

$$
\frac{\partial \hat{\eta}}{\partial Z}=\sum_{k=1}^{K} \hat{\beta}_{k} k Z^{(k-1)}=0
$$

This optimum is located empirically by finding the $z$-value in the data, $\tilde{Z}$, which falls at the minimum predicted logit within a suitable range of $z$-values (i.e., $-4,4$ ), or $\tilde{Z}=Z[\hat{\eta}=\min (\hat{\eta})]$ as shown in Figure A1. These optima compare favorably to the empirical optima determined using the procedures of Solis et al. (2000), in which log IMR is plotted for standardized birth weight values in a specific gestational age range. The empirical IMRs (shown as points) are based on aggregating the data over $250 \mathrm{~g}$ intervals of birth weight in the 37-42 week range.

Figure A1 shows the observed and fitted logits by $Z$ from polynomial models fit to white, black, and Mexican-American mortality for births occurring between 37 and 42 weeks gestation. It is clear that the optimum of one standard deviation above the gestational age-specific mean birth weight slightly overestimates the survival optimum for Mexican Americans, but appears to be a reasonable assumption for blacks and whites in the 37-42 week gestational age range. As might be expected, for gestations less than 37 weeks, the optima are further from the mean at 1.99sd, 1.76sd, and 1.79sd above gestational age-specific mean for whites, blacks, and Mexican Americans, respectively. For gestations greater than 42 weeks, the optima are somewhat closer to the mean: whites $(0.93 \mathrm{sd})$, blacks (1.03sd), and Mexican Americans (0.40sd). These results differ from those of Adams et al. (1991) who find that the optimum birth weight for blacks is closer to the mean that it is for whites. However, they focus on perinatal mortality and include males in the analysis. Table A1 provides more information on the particular standardization that would apply to each gestational age by race category for our sample. We present the mean, standard deviation, percentage of births occurring in the 22-47 week gestational age range for whites, blacks, and Mexican Americans, respectively.

\section{Further results on age-varying effects}

In this section we discuss in more detail the results from a set of simpler models using both age-invariant and age-varying effects of birth weight (small and heavy) and gestational age (early and late), in addition to an age-invariant interaction effect of small $\times$ early, to show that specifying age-varying effects of small birth weight and 
Figure A1: Observed and fitted logits by gestational age standardized birth weight $(Z)$ for Whites, Blacks, and Mexican Americans: 37-42 weeks of gestation.

White

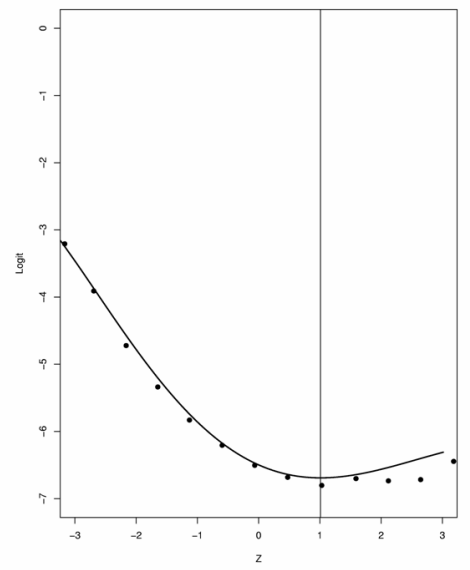

Black

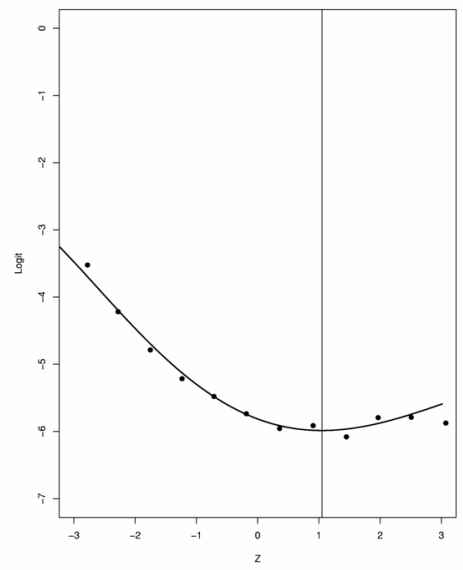

Mexican American

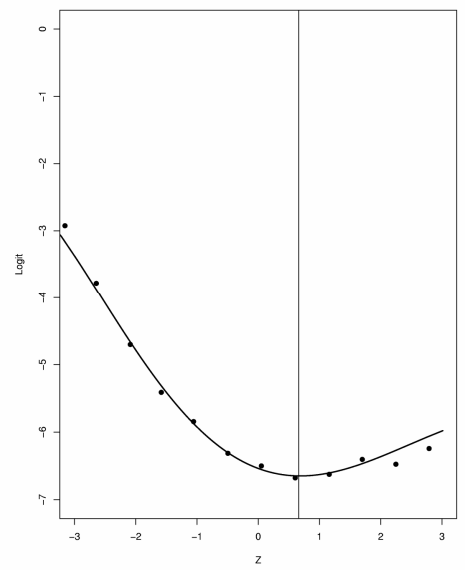

Whites: The lowest mortality is 1.01 standard deviations above the gestational age-specific mean birth weight.

Blacks: The lowest mortality is at a point 1.05 standard deviations above the gestational age specific mean birth weight.

Mexican-Americans: The lowest mortality is at a point 0.66 standard deviations above the gestational age-specific mean birth weight. 
Powers et al.: Race/Ethnic differences and age-variation

Table A1: Gestational-age specific parameters of the birth weight distribution U.S. females 1995-1998

\begin{tabular}{|c|c|c|c|c|c|c|c|c|c|}
\hline \multirow{3}{*}{$\begin{array}{l}\text { Gestational } \\
\text { Age } \\
\text { (Weeks) }\end{array}$} & \multicolumn{3}{|l|}{ White } & \multicolumn{3}{|l|}{ Black } & \multicolumn{3}{|c|}{ Mexican American } \\
\hline & \multicolumn{2}{|c|}{ Birth Weight (g) } & \multirow[t]{2}{*}{ Pct. } & \multicolumn{2}{|c|}{ Birth Weight (g) } & \multirow[t]{2}{*}{ Pct. } & \multicolumn{2}{|c|}{ Birth Weight (g) } & \multirow[t]{2}{*}{ Pct. } \\
\hline & Mean & Std. Dev. & & Mean & Std. D & & Mean & Std. D & \\
\hline 22 & 714.4 & 325.7 & 0.01 & 761.4 & 348.7 & 0.07 & 752.0 & 338.4 & 0.02 \\
\hline 23 & 686.9 & 264.3 & 0.03 & 722.2 & 305.3 & 0.12 & 718.6 & 278.2 & 0.03 \\
\hline 24 & 908.4 & 617.2 & 0.04 & 953.0 & 642.4 & 0.18 & 953.5 & 644.2 & 0.05 \\
\hline 25 & 990.9 & 616.0 & 0.05 & 1030.5 & 640.4 & 0.22 & 1078.1 & 681.7 & 0.06 \\
\hline 26 & 1090.5 & 615.8 & 0.07 & 1155.3 & 653.6 & 0.27 & 1199.3 & 686.3 & 0.08 \\
\hline 27 & 1186.6 & 597.0 & 0.08 & 1256.4 & 642.3 & 0.28 & 1267.4 & 624.7 & 0.09 \\
\hline 28 & 1597.8 & 934.1 & 0.11 & 1605.7 & 879.1 & 0.38 & 1815.6 & 993.0 & 0.13 \\
\hline 29 & 1809.6 & 927.3 & 0.14 & 1788.9 & 864.3 & 0.42 & 2060.2 & 956.5 & 0.16 \\
\hline 30 & 1999.1 & 900.6 & 0.19 & 1992.3 & 830.0 & 0.61 & 2247.4 & 912.2 & 0.24 \\
\hline 31 & 2115.1 & 833.5 & 0.25 & 2120.7 & 786.0 & 0.73 & 2381.3 & 846.0 & 0.34 \\
\hline 32 & 2288.9 & 779.4 & 0.35 & 2275.0 & 726.3 & 0.95 & 2548.6 & 811.1 & 0.45 \\
\hline 33 & 2437.9 & 707.0 & 0.52 & 2430.4 & 666.3 & 1.31 & 2693.0 & 711.6 & 0.69 \\
\hline 34 & 2615.1 & 643.2 & 0.93 & 2579.9 & 627.1 & 2.10 & 2850.2 & 653.0 & 1.29 \\
\hline 35 & 2785.5 & 577.3 & 1.67 & 2719.5 & 568.6 & 3.20 & 2954.5 & 592.2 & 2.14 \\
\hline 36 & 2931.0 & 516.1 & 3.19 & 2823.2 & 513.1 & 4.91 & 3018.0 & 531.8 & 3.47 \\
\hline 37 & 3096.6 & 476.8 & 6.66 & 2956.6 & 470.8 & 8.53 & 3118.2 & 477.7 & 6.84 \\
\hline 38 & 3269.2 & 453.4 & 14.56 & 3096.5 & 450.5 & 15.31 & 3242.7 & 444.9 & 14.69 \\
\hline 39 & 3399.7 & 441.3 & 24.09 & 3211.7 & 440.9 & 21.84 & 3350.5 & 433.8 & 23.92 \\
\hline 40 & 3493.2 & 439.4 & 24.44 & 3296.6 & 444.5 & 19.94 & 3429.7 & 432.5 & 23.40 \\
\hline 41 & 3551.5 & 449.8 & 13.81 & 3350.7 & 455.8 & 10.46 & 3479.7 & 442.2 & 12.67 \\
\hline 42 & 3523.0 & 470.3 & 4.55 & 3300.8 & 472.1 & 3.95 & 3454.9 & 460.7 & 4.54 \\
\hline 43 & 3466.1 & 467.4 & 2.05 & 3253.8 & 473.3 & 2.04 & 3420.8 & 455.9 & 2.25 \\
\hline 44 & 3464.1 & 472.1 & 1.12 & 3272.8 & 465.3 & 1.12 & 3440.2 & 451.1 & 1.24 \\
\hline 45 & 3466.9 & 478.4 & 0.60 & 3277.6 & 474.4 & 0.59 & 3454.0 & 462.3 & 0.67 \\
\hline 46 & 3460.1 & 489.3 & 0.31 & 3268.0 & 496.2 & 0.30 & 3454.4 & 468.2 & 0.34 \\
\hline 47 & 3431.1 & 488.6 & 0.18 & 3210.2 & 492.1 & 0.18 & 3418.9 & 488.2 & 0.21 \\
\hline
\end{tabular}

Source : 1995-1998 NCHS linked birth/infant death files. 
early and late gestational age statistically accounts for the black mortality disadvantage within the first week of life. We fit several models that include mixtures of ageinvariant and age-varying effects of birth outcomes. Each model includes an ageinvariant early $\times$ small interaction effect, plus age-invariant or age-varying effects of early, late, small, and heavy. Table A2 shows the estimated black/white hazard ratios corresponding to several models (compare to Tables 1 and 2). The presence of an agevarying effect for a selected variable is indicated by a shaded cell, otherwise the modeled effect is age-invariant. Results are listed roughly according to the magnitude of the black/white hazard ratios. From Table A2, we find that the greatest relative black early neonatal mortality disadvantage is evident from the model without age-varying effects. Similarly, we find that fitting age-varying effects for all birth outcomes results in a slight black neonatal survival advantage. A model with age-varying effects for early, late, and small with an age-invariant effect of heavy, yields near equity in black/white neonatal survival advantage.

Table A2: $\quad$ Black/White hazard ratios corresponding to the first week of life (selected models)

\begin{tabular}{|l|l|l|l|l|l|}
\hline \hline \multirow{2}{*}{$\begin{array}{l}\text { Black/White Hazard Ratio* } \\
\text { in the First Week of Life }\end{array}$} & Early & Late & Small & \multirow{2}{*}{ Heavy } \\
\cline { 2 - 6 } \multicolumn{2}{|l|}{} & \multicolumn{3}{|l|}{ Age-Varying Effect (shaded) } \\
\hline Day [0 - 1) & 2.073 & & & & \\
Day [1-7) & 1.809 & & & & \\
\hline Day [0-1) & 1.773 & & & & \\
Day [1-7) & 1.549 & & & & \\
\hline Day [0-1) & 1.329 & & & & \\
Day [1-7) & 1.405 & & & & \\
\hline Day [0-1) & 1.324 & & & & \\
Day [1-7) & 1.420 & & & & \\
\hline Day [0-1) & 1.043 & & & & \\
Day [1-7) & 1.068 & & & & \\
\hline Day [0-1) & 0.957 & & & & \\
Day [1-7) & 1.074 & & & & \\
\hline
\end{tabular}

Source: 1995-1998 NCHS linked birth/infant death files

*Models include age invariant early $\times$ small interaction effect plus age-varying or age-invariant effects of early, late, small, and heavy, in addition to baseline hazard terms corresponding to 7-27 days and the postneonatal period. 
Powers et al.: Race/Ethnic differences and age-variation 